\title{
Entropy production in high energy collisions
}

\author{
G. Bertsch \\ Physics Department, Michigan State University, East Lansing, Michigan 48824 \\ and Institute for Theoretical Physics, University of California, \\ Santa Barbara, California 93106 \\ J. Cugnon \\ Institut de Physique, Université de Lìge, Liege, Belgium
}

(Received 29 June 1981)

\begin{abstract}
The entropy production in high-energy collisions is computed in a Monte Carlo cascade model. For collisions of ${ }^{40} \mathrm{Ca}$ on ${ }^{40} \mathrm{Ca}$ at $800 \mathrm{MeV} /$ nucleon beam energy, the computed entropy is 4.4 per particle, about a unit higher than estimated from bulk dynamics. The particle correlation function of the final state is also computed, and is found to be in reasonable accord with a thermal distribution of the same entropy. With such low entropy values, most of the particles emerge in clusters, contrary to experiment. Thus the cascade calculation supports the conclusion of Siemens and Kapusta, that additional degrees of freedom become accessible in heavy ion collisions, beyond those in a conventional nuclear description.
\end{abstract}

$\left[\begin{array}{c}\text { NUCLEAR REACTIONS }{ }^{40} \mathrm{Ca}\left({ }^{40} \mathrm{Ca} \text {, light composites), } E=800\right. \\ \mathrm{MeV} / \text { nucleon; calculated entropy, deuteron production, final state } \\ \text { clustering. }\end{array}\right]$

\section{INTRODUCTION}

There is a close connection between deuteron production in high energy heavy ion collisions and the entropy generated in the collisions, pointed out by Siemens and Kapusta. ${ }^{1}$ These authors also made the provocative claim that the generated entropy is larger than can be understood with conventional models of the dynamics of nuclear matter. However, this result relies on the validity of a macroscopic treatment of the dynamics, ignoring size effects due to the finite mean free path of the nucleons. In this paper we study the entropy generation and deuteron formation in a finite system using a Monte Carlo cascade model. We do find that additional entropy is produced in a finite system, compared to the bulk value. Nevertheless, the predicted entropy is much lower than the empirical data on deuteron emission suggests.

We begin with the definition of entropy in a system of noninteracting fermions,

$$
S=-\int d \gamma[f \ln f+(1-f) \ln (1-f)] .
$$

Here $f$ is the occupation probability of phase space, and $d \gamma$ is the phase space volume element,

$$
\int d \gamma=4 \int \frac{d^{3} r d^{3} p}{(2 \pi \hbar)^{3}}
$$

The spin-isospin degrees of freedom give rise to the factor of 4 in Eq. (2). We shall consider only the dilute limit of Eq. (1), $f<<1$, which will be justified later. Then $\ln (1-f)$ may be expanded and Eq. (1) reduces to the classical equation for the entropy,

$$
S=A-\int d \gamma \ln f=A(1-\langle\ln f\rangle)
$$

where $A$ is the number of particles. As a practical matter, $f$ will be computed in the cascade model by counting particles in cells of phase space. The optimum choice of cell configuration requires some numerical analysis, which we discuss in Sec. II. The heavy ion collision we consider is ${ }^{40} \mathrm{Ca}$ on ${ }^{40} \mathrm{Ca}$ at $800 \mathrm{MeV}$ per nucleon bombarding energy. The actual details of the entropy computation are presented in Sec. III. For the cascade calculations, 
we have used the model described in Ref. 2. We used the computer results of Ref. 2 together with an independently written computer program based on the same cross sections. We will not describe the cascade aspect of the calculations, except to note that pions are included via $\Delta$ excitations of nucleons. The $\Delta$ 's are assumed to have decayed to pions and nucleons when the nucleon distribution function is calculated. We will only examine the entropy associated with the nucleus; there is of course additional entropy associated with the pions in the final state. In Sec. IV we discuss the relation between the entropy, the average phase space density, and the pair correlation function. The cascade results are found to be in reasonable accord with approximations based on thermal equilibrium. Finally, we confirm the finding of Ref. 1 that too many deuterons are predicted, compared to what is observed.

\section{NUMERICAL ANALYSIS}

To calculate the distribution function $f$ in the cascade model, we first divide the phase space into cells, labeled by index $i$. The number of particles $n_{i}$ in each cell is found at some fixed time in the cascade. Statistics can be improved by summing $n_{i}$ over many runs of the cascade program. The distribution function $f$ is then estimated as

$$
\bar{f}_{i}=\frac{n_{i}}{R \int_{i} d \gamma},
$$

where $R$ is the number of runs of the cascade. The division of phase space into cells must be done with some care. Ideally the cells should be small enough so that $f$ is uniform within the sixdimensional volume of the cell. Thus the cell division shown in Fig. 1(a) would underestimate $f$ and give too high an entropy. Conflicting with the demand for small cell size is the requirement that the cells include enough particles so that statistical errors in the numbers $n_{i}$ do not become significant. An extreme case of this is shown in Fig. 1(b). Fortunately, the entropy is such a smooth function of $f$ that there is a wide tolerance on these requirements.

The effect of nonuniformity of $f$ within the cells can be examined with a simple model problem. Suppose there is some phase space variable $z \geq 0$, with the distribution given by

$$
f=f_{0} e^{-z / z_{0}} \text {. }
$$
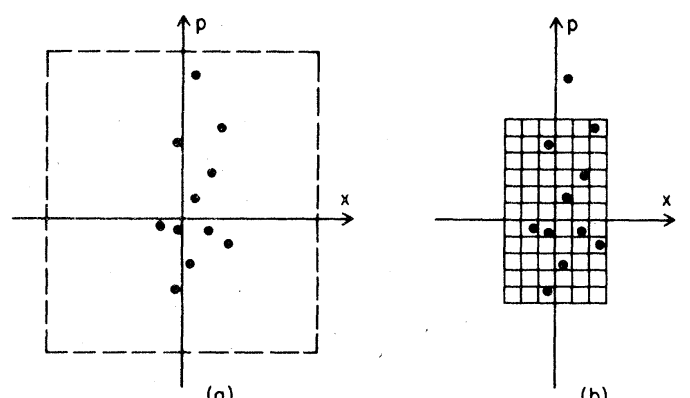

FIG. 1. Phase space density estimated by counting particles in cells. In (a) the cell is too large, and the density will be underestimated. In (b) the cells are too small, with the opposite effect.

Then Eq. (3) yields an entropy

$$
S / A=\left(2-\ln f_{0}\right) \text {. }
$$

We compare this result with the estimate obtained by dividing $z$ into intervals of length $\Delta z=\xi z_{0}$. This result is

$$
\begin{aligned}
\left.\frac{S}{A}\right|_{\mathrm{est}} & =1-\sum_{i} \frac{\Delta z \bar{f}_{i} \ln \bar{f}_{i}}{\sum_{i} \Delta z \bar{f}_{i}} \\
& =1-\ln \left(1-e^{-\xi}\right) f_{0} / \xi+\xi /\left(e^{\xi}-1\right) .
\end{aligned}
$$

For $\xi=1$, corresponding to a ratio of particles $e$ in adjacent intervals, the difference between (6) and (7) is 0.04 , i.e., the error in the entropy is only 0.04 per particle. Thus if we aim to compute the entropy to an accuracy of $\sim 0.1$, we can permit cell configurations that have $f$ varying by a factor of 3 or so between adjacent cells. It is necessary that the cell boundary allow all the variations in $f$ to be displayed, but only at this crude level.

The next question is the error due to counting statistics with a finite number of particles. There is a systematic bias in estimates based on Eq. (4) with $n_{i}$ small. This is illustrated by the cell configuration of Fig. 1(b), having most cells empty. The density in cells containing particles is then estimated too high. Since only occupied cells contribute in the calculation of $\sum_{i} \bar{f}_{i} \ln \bar{f}_{i}$, the computed entropy would be low. It is straightforward to determine this bias, treating the fluctuations in $n_{i}$ about the mean $\left\langle n_{i}\right\rangle$ with Poisson statistics. The average deviation of the estimate from the true cellular entropy is 


$$
\begin{aligned}
\left.\frac{\Delta S}{A}\right|_{\text {statistical }} & =\frac{\left\langle\sum_{i}\left(\left\langle n_{i}\right\rangle \ln \left\langle n_{i}\right\rangle-n_{i} \ln n_{i}\right)\right\rangle}{\sum_{i} n_{i}} \\
& =-\frac{\left\langle 2 \sum_{i} n_{i} \ln \left(1+\frac{\delta n_{i}}{\left\langle n_{i}\right\rangle}\right)\right\rangle}{\sum_{i} n_{i}}
\end{aligned}
$$

where $\delta n_{i}=n_{i}-\left\langle n_{i}\right\rangle$. We expand the logarithm to second order and use $\left\langle\delta n_{i}{ }^{2}\right\rangle=\left\langle n_{i}\right\rangle$, to evaluate (8) as

$$
\left.\frac{\Delta S}{A}\right|_{\text {statistical }} \simeq-\frac{\sum_{i} \frac{1}{2}}{\sum_{i} n_{i}}=-\frac{1}{2} \frac{C}{R A} .
$$

Here $C$ is the number of occupied cells and $R A$ is the total number of particles in $R$ runs. Naively we might have expected to require a high accuracy in each cell but Eq. (9) is much less demanding. For example, to achieve an accuracy of 0.1 , we need only an average of 5 particles in each occupied cell. Besides this statistical bias, there will be a statistical uncertainty due to the fluctuations in the computed $S$ about the mean. We will not attempt to evaluate this analytically, but will be content to determine this error "experimentally" by generating an ensemble of computed entropies.

\section{PRACTICAL DETAILS}

The distribution function $f$ depends on six variables and it would not be practical to make a brute force division into cells in all six dimensions. We have to select the variables to which $f$ is most sensitive and ignore the others. We may start from the variables in polar coordinates: the momentum $\overrightarrow{\mathrm{p}}$ specified by its magnitude $p$, its polar angle $\theta_{p}$ and azimuthal angle $\phi_{p}$; the magnitude $r$ and its direction relative to $p$ specified by polar angle $\theta_{p r}$ and azimuthal angle $\phi_{p r}$. Obviously, the dependence of $f$ on $r$ and $p$ needs to be treated explicitly. We will limit our calculation to head-on collisions, so that $\phi_{p}$ will be unnecessary. The remaining variables $\theta_{p r}, \theta_{p}$, and $\phi_{p r}$ will be considered in turn.

The cascade model describes ${ }^{40} \mathrm{Ca}$ on ${ }^{40} \mathrm{Ca}$ at 800 $\mathrm{MeV}$ per nucleon bombarding energy. The particle numbers are accumulated over 10 runs of the program, giving 800 particles all together. We examine the distribution at a time $15 \mathrm{fm} / c$ after the nuclei touch. By this time the $N-N$ collisions have essentially ceased. We first look at the distribution function in $r$ and $p$ alone, dividing phase space up into spherical shells with $\Delta r=2 \mathrm{fm}$ and $\Delta p=0.2$ $\mathrm{GeV} / c$. Table I shows the particle numbers $n_{i}$ for this cell configuration. We only count particles that have been struck at least once, so the number of particles in the table (776) is less than 800 . Notice that the particles with large $r$ tend to have large $p$. At $t=15 \mathrm{fm} / c$ the system is a nearly spherical expanding gas, and there are obviously going to be correlations between the directions of $p$ and $r$ as well. Accordingly, we subdivide the cells depending on $\theta_{p r}$. Table II shows the $n_{i}$ with the subdivision into three, using $\theta_{p r}=45^{\circ}$ and $18^{\circ}$ as boundaries. Notice that the particles with largest $r$ tend to be in the cell with smallest angle $\theta_{p r}$. The entropy computed without the $\theta_{p r}$ division is 5.5 per particle, and with the division it is 4.5. Clearly it is essential to treat this correlation. A convenient way to treat the $\theta_{p r}$ correlation without using addition cell subdivisions is to shift the origin in the momentum space shells. The momentum vector is redefined

$$
\overrightarrow{\mathrm{p}}^{\prime}=\overrightarrow{\mathrm{p}}-c \overrightarrow{\mathrm{r}}
$$

The parameter $c$ is chosen to reduce the residual

TABLE I. Nucleon distribution in $p$ and $r$ for ${ }^{40} \mathrm{Ca}+{ }^{40} \mathrm{Ca}$ collisions at $800 \mathrm{MeV} /$ nucleon, summed over ten collisions. The shell thickness of the cells is $\Delta p=0.2 \mathrm{GeV} / c$ and $\Delta r=2$ fm.

\begin{tabular}{lrrrrrr}
\hline$r(\mathrm{fm})$ & $p(\mathrm{GeV} / \mathrm{c})$ & $0.0-0.2$ & $0.2-0.4$ & $0.4-0.6$ & $0.6-0.8$ & $0.8-1.0$ \\
\hline $0-2$ & 10 & 35 & 32 & 16 & 4 \\
$2-4$ & 25 & 82 & 107 & 50 & 12 \\
$4-6$ & 5 & 47 & 88 & 93 & 22 \\
$6-8$ & 0 & 6 & 31 & 67 & 22 \\
$8-10$ & 0 & 0 & 1 & 10 & 11 \\
\hline
\end{tabular}


TABLE II. Nucleon distribution in $p$ and $r$, as in Table I, divided according to $\theta_{p r}$.

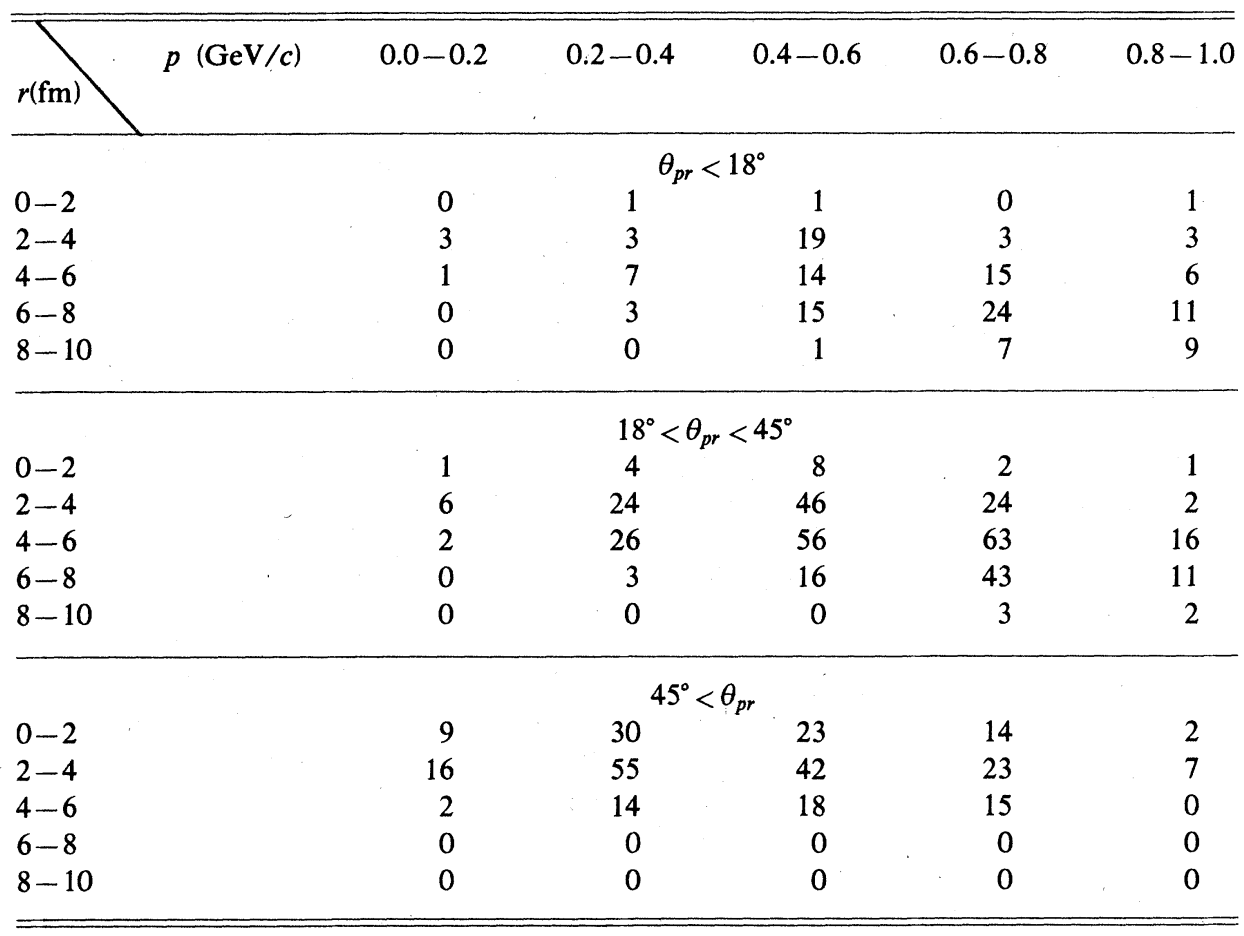

$\theta_{p r}$ correlation. In Table III we display the number distribution with $c=0.07 \mathrm{GeV} / c \mathrm{fm}$, divided according to $\theta_{p r}$ greater than or less than $90^{\circ}$. The entropy without this $\theta_{p r}$ division is computed to be 4.40 per particle; with the division it is only reduced by 0.03 units. Thus we can drop the $\theta_{p r}$ dependence, once the transformation of coordinates has been made.
We next consider the dependence on $\theta_{p}$. A subdivision of the cells using $\left|\cos \theta_{p}\right|=0.5$ as a boundary lowers the computed entropy per particle by 0.04 units, i.e., there is no perceptible dependence on $\theta_{p}$. This is to be expected with the cascade model of Ref. 2. The authors found that the central collisions resemble a fireball, with the momentum distributed more or less equally in all

TABLE III. Nucleon distribution, as in Table I, with shifted momentum origin accoräing to Eq. (10).

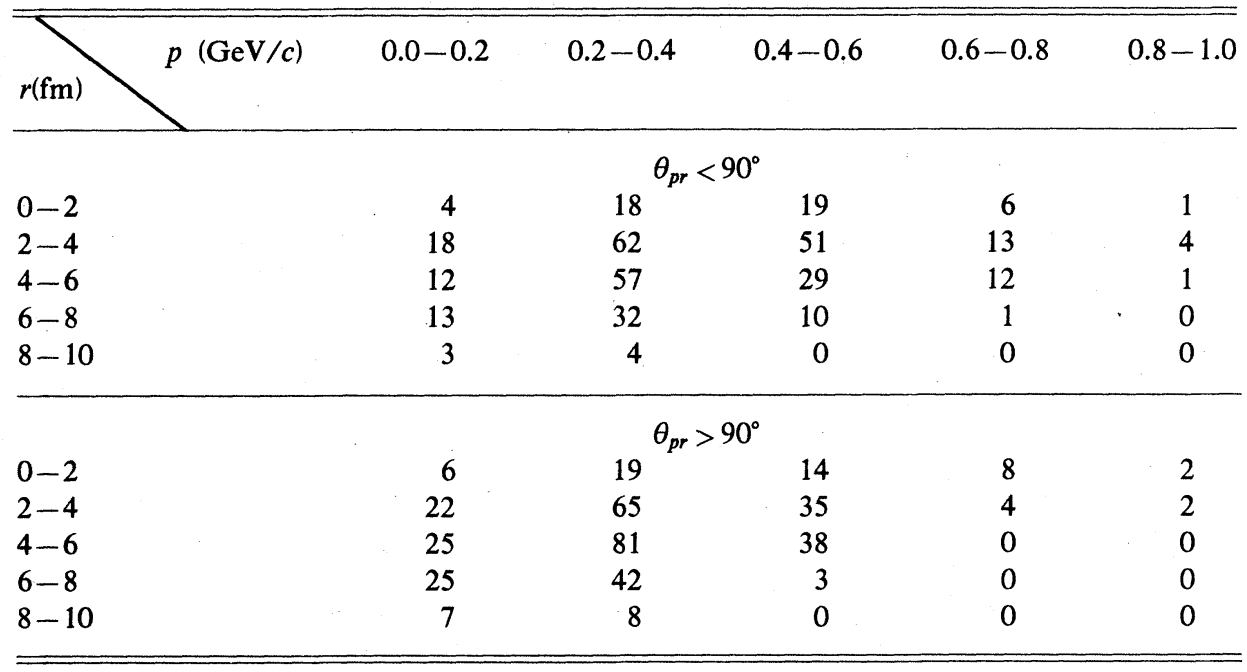


directions. For completeness we also checked the dependence on $\theta_{r}$, and found a negligible change in $S, \Delta S / A=0.02$. The statistical fluctuations in $S / A$ appear to be very small, with three independent sets of runs giving $S=4.41,4.41$, and 4.40 .

Having found an appropriate cell configuration to compute the entropy, we now examine $S$ as a function of time. Initially, the particles have zero entropy in the ground states of the nuclei, but we cannot handle $S$ in the quantum regime. We therefore only consider particles that have been struck in $N N$ collisions in computing $\bar{f}_{1}$. Figure 2 shows how the entropy of the struck particles evolves with time. At the very beginning phase space is sparsely populated and the specific entropy is high. As time goes on the development becomes a steady process and the entropy approaches 4.3 per particle. Once the collisions have ceased, the flow of $f$ in phase space is incompressible and $S$ should not change. In fact the computed $S$ increases by less than 0.1 from $t=15$ to $t=22$, showing the inherent accuracy of the computation.

\section{FROM BULK DYNAMICS TO DEUTERON PRODUCTION}

There is a large difference between the entropy we have calculated and the predicted entropy from bulk dynamics, which we would like to understand better. The maximum density reached in the collision is about four times nuclear matter density. ${ }^{2}$ In thermal equilibrium at this density, the entropy

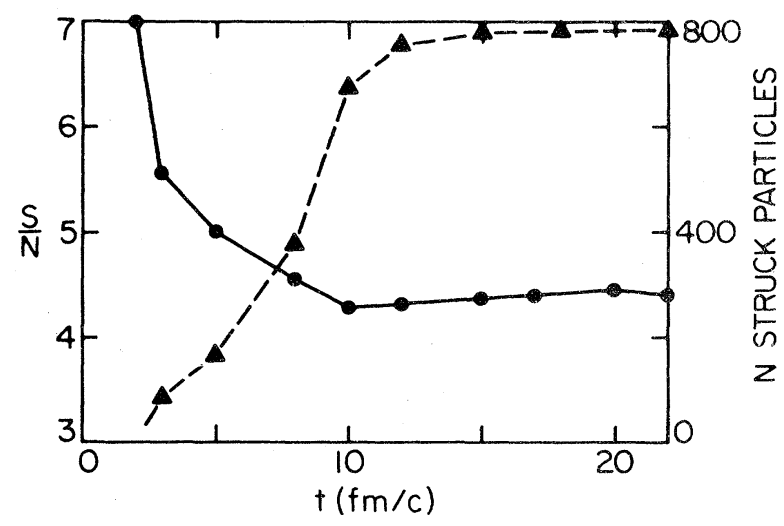

FIG. 2. Time evloution of entropy for collisions of ${ }^{40} \mathrm{Ca}$ on ${ }^{40} \mathrm{Ca}$ at $800 \mathrm{MeV} /$ nucleon. Also shown (triangles) is the number of particles that have undergone collisions as a function of time. Nearly all the particles have collided by $12 \mathrm{fm} / c$, and the collisions essentially cease after $15 \mathrm{fm} / c$. would be 3.2 per nucleon, compared to the 4.4 we calculated. The first thing we note is that the average density is much less than the maximum. At $t=8 \mathrm{fm} / c$, the time maximum density is acheived, the average density is only twice nuclear matter density. Here a thermal distribution would give $S / A=3.8$. The remaining half unit of entropy can possibly be understood as arising from the finite mean free path of the nucleons. If we study $f(p, r)$ for fixed $p$ different from the initial momentum, there must be a smearing in the $r$ dependence by a distance of the order of the mean free path. Thus the effective volume of the system is larger by a dimension of the order of the mean free path.

We now turn to the questions of correlation functions and deuteron formation, and their relation to the entropy and the single-particle distribution function. If the system is in chemical equilibrium at low density, then the relation between entropy and deuteron abundance can be derived from the Sackur-Tetrode equation. With the temperature large compared to the deuteron binding energy, this is

$$
\frac{S}{A}=\frac{5}{2}+\frac{3}{2} \ln 2-\ln \left(2 N_{d} / 3 N_{p}^{\text {free }}\right) .
$$

Here $N_{d}$ is the number of deuterons produced and $N_{p}^{\text {free }}$ is the number of free protons. However, the conditions in a heavy ion collision do not obviously satisfy the low density assumption. The $N-N$ collisions cease at $\sim \frac{1}{2}$ nuclear matter density, too high to neglect the interaction between chemical species. A formula with identical appearance to Eq. (11) can be derived with assumptions other than low density. The first step in the argument is to note that the deuteron abundance is related to the two-particle correlation function by ${ }^{1}$

$$
N_{d}=3 \int \frac{d^{3} r d^{3} p}{(2 \pi \hbar)^{3}} g_{d}(r, p) g_{n p}(r, p) \text {. }
$$

Here $g_{d}$ is the deuteron density matrix, and $g_{n p}$ is the correlation function between neutrons and protons of specific spin orientations,

$$
\begin{aligned}
& g_{n p}\left(r_{12}, p_{12}\right) \\
& \quad=\int \frac{d^{3} R d^{3} P}{(2 \pi \hbar)^{3}} f^{(2)}\left(r_{n}, p_{n} ; r_{p}, p_{p}\right),
\end{aligned}
$$

where $r_{12}=r_{n}-r_{p}, p_{12}=\left(p_{n}-p_{p}\right) / 2, R=\left(r_{n}\right.$ $\left.+r_{p}\right) / 2$, and $P=p_{n}+p_{p}$. If we assume that $g_{n p}$ is uniform over a sufficiently large region of phase space, we may use the normalization condition of $g_{d}$ to replace Eq. (12) by 
TABLE IV. The pair correlation function $g_{n p}(r, p)$ near $(0,0)$.

\begin{tabular}{|c|c|c|c|c|}
\hline$p_{12}=\left(p_{n}-p_{p}\right) / 2 \mathrm{GeV} / c$ & $0.0-0.1$ & $0.1-0.2$ & $0.2-0.3$ & $0.3-0.4$ \\
\hline $0-1$ & 15.1 & 7.8 & 4.9 & 0.6 \\
\hline $1-2$ & 12.1 & 6.7 & 3.3 & \\
\hline $2-3$ & 8.8 & 5.8 & & \\
\hline $3-4$ & 6.4 & & & \\
\hline
\end{tabular}

$$
N_{d} \simeq 3 g_{n p}(0,0) .
$$

We also assume that the two-particle distribution function is given by the product of single-particle functions, $f_{n p}^{(2)}=f_{n} f_{p}$. Then $g_{n p}(0,0)$ can be evaluated from Eq. (13) as

$$
\begin{aligned}
g_{n p}(0,0) & =\int d \gamma f_{p}(r, p / 2) f_{n}(r, p / 2) \\
& =8 N_{p \uparrow}\left\langle f_{n}\right\rangle,
\end{aligned}
$$

where $N_{p \uparrow}=N_{p} / 2$ is the total number of protons with given spin orientation. We also need to invoke the relation between $S$ and $\langle f\rangle$ for a system in thermal equilibrium,

$$
\frac{S}{A}=1+\frac{3}{2}(1-\ln 2)-\ln \langle f\rangle .
$$

Equations (14) - (16) can be combined to give an equation with identical appearance to Eq. (12). However, the interpretation of the particle numbers are different. In Eq. (14) $N_{d}$ is the number of deuteronlike pairs, including pairs contained in larger clusters. Similarly $N_{p}$ in the high density derivation is the total number of protons in the system, including bound protons.

The assumptions in Eq. (14) and (16) can be tested with the cascade results. Let us first consider the relation between $\langle f\rangle$ and $S$. Computing $\langle f\rangle$ as $\left\langle\bar{f}_{i}\right\rangle$, the ${ }^{40} \mathrm{Ca}+{ }^{40} \mathrm{Ca}$ cascade yields

$$
\langle f\rangle \simeq 0.07 \text {. }
$$

Alternatively, $\langle f\rangle$ can be calculated from the two-particle correlation function via Eq. (15). We estimate the correlation function by counting pairs

TABLE V. Average occupation probability $\langle f\rangle$ computed various ways.

\begin{tabular}{lr}
\hline & $\langle f\rangle$ \\
\hline Direct cell average, Eq. (17) & 0.07 \\
Eq. (15) & 0.09 \\
Eq. (16) & 0.05 \\
\hline \hline
\end{tabular}

in cells of $r_{12}$ and $p_{12}$, summing over $R$ and $P$,

$$
\bar{g}_{n p}=\frac{n_{i}}{\int_{i} \frac{d^{3} r_{12} d^{3} p_{12}}{(2 \pi \hbar)^{3}}}
$$

The computed $\bar{g}_{n p}$ is shown in Table IV for cells in the form of spherical shells with $\Delta p=0.1 \mathrm{GeV} / c$ and $\Delta r=1 \mathrm{fm}$. At the moment we are just interested in $g_{n p}(0,0)$, which we estimate by the entry in the first cell, $0 \leq r \leq 1 \mathrm{fm}, 0 \leq p \leq 0.1 \mathrm{GeV} / c$. Then $\langle f\rangle$ can be found from Eq. (15), and we find the value shown in the second entry of Table V. If the distribution were thermal, Eq. (16) could be applied yielding the value for $\langle f\rangle$ shown in the third entry to Table $V$. The thermal value is less than the other estimates. We conclude that the actual distribution function is less homogeneous than a thermal distribution, and there will be more clusters than predicted from the entropy of a thermal distribution.

We next examine the validity of the approximation leading to Eq. (14), that $g_{n p}$ is uniform. Comparing the first two cells in $r$ in Table IV, we see that $\bar{g}$ is reasonably uniform on the spatial scale of the deuteron. This is not the case for the $p$ dependence. At small $r_{12}$, the $p_{12}$ dependence can be characterized by a temperature, $T \simeq 25 \mathrm{MeV}$.

TABLE VI. Pair clustering in final state, $N_{d} / N_{p}$.

\begin{tabular}{lc}
\hline \multicolumn{1}{c}{ Method } & $N_{d} / N_{p}$ \\
\hline $\begin{array}{l}\text { Eq. (14), with } g_{n p}(0,0) \\
\text { from Eqs. (15) and (17). }\end{array}$ & 0.8 \\
$\begin{array}{l}\text { Eq. (14), with } g_{n p}(0,0)=15.1 \\
\text { from Table IV }\end{array}$ \\
$\begin{array}{l}\text { Eq. (12), with } \\
g_{d}(r, p) \cong \theta(0.2-p) \theta(2.0-r)\end{array}$ \\
Experiment
\end{tabular}


TABLE VII. Cross sections for particle production in $\mathrm{Ar}+\mathrm{KCl}$ collisions at 800 $\mathrm{MeV} /$ nucleon, from Ref. 4.

\begin{tabular}{cccccc}
\hline Product & $p$ & $d$ & ${ }^{3} \mathrm{H}$ & ${ }^{3} \mathrm{He}$ & $\alpha$ \\
\hline$\sigma$ (barns) & 14.1 & 4.0 & 0.62 & 0.52 & 0.16 \\
deuteron clusters & 0 & 1 & $\frac{3}{2}$ & $\frac{3}{2}$ & 3 \\
$\sigma_{\boldsymbol{d}}$ & 0 & 4.0 & 0.9 & 0.8 & 0.5 \\
\hline
\end{tabular}

Parenthetically, this is a good illustration of the cooling of a system in isentropic expansion. ${ }^{3}$ In any case, the falloff in $p$ is significant over the scale of the momentum in the deuteron density matrix $p \sim 0.2 \mathrm{GeV} / c$. We thus cannot affirm the assumption underlying Eq. (14). The various possible assumptions lead to different predictions for the clustering in the final state, and we compare these in Table VI. Remember that $N_{d} / N_{p}$ is the ratio of deuteronlike pairs in composite particles to the total number of protons. We see that a major fraction of the protons are predicted to be found in clusters.

Experimentally, far less clustering is found. Nagamiya et $a l .{ }^{4}$ have measured the particle abundances emerging from $\mathrm{Ar}+\mathrm{KCl}$ collisions at 800 $\mathrm{MeV} / \mathrm{u}$. These are quoted in Table VII. Let us ask for the number of protons clustered in deuterons or in deuteronlike pairs in heavier nuclei, relative to the total number of protons observed. The number of deuteronlike pairs in the heavier composite nuclei are shown in the second line of the table. The partial cross sections for these deuteronlike pairs is shown in the third line, and the total cross section for "deuterons" is $6 \mathrm{~b}$, to be compared with $20 \mathrm{~b}$ for all protons, including bound ones. The ratio of these cross sections is shown at the bottom of Table VI. We see that clusters are a much smaller fraction of the total than predicted with the cascade model. Assuming that the cascade results do not change substantially at nonzero impact parameter, the conclusion follows that there is more disorder produced in the final state than is compatible with a description based on independent nucleon collisions.

\section{ACKNOWLEDGMENTS}

G.F.B. is grateful for many helpful discussions with M. Gyulassy, E. Remler, and P. Siemens, ana acknowledges support of the National Science Foundation under Grant PHY-77-27084. We also thank S. Nagamiya for communicating his experimental data prior to publication.
${ }^{1}$ P. Siemens and J. Kapusta, Phys. Rev. Lett. $\underline{43}, 1486$ (1979).

2J. Cugnon, T. Mizutani, and J. Vandermeulen, Nucl. Phys. A352, 505 (1981).
3P. Siemens and J. Rasmussen, Phys. Rev. Lett. 42, 880 (1979).

${ }^{4} \mathrm{~S}$. Nagamiya et al., Lawrence Berkeley Laboratory Report LBL 12123, 1980; see Table IV. 